\title{
TÚ Y YO. LECTURA DE UN «SONETO DE ARTIFICIO» DE BERNARDO SCHIAVETTA
}

\author{
Jan Baetens
}

Universiteit Maastricht

\section{ELOGIO DE LO SIMPLE}

La literatura hiperconstruida, gustosamente artificiosa, infinitamente cincelada, a menudo pasa por ser secreta, obstrusa y hasta hermética, como si sólo pudieran acceder a ella un círculo restringido de iniciados. Sobre todo cuando invoca explícitamente a autores tales como Marino, Góngora o Mallarmé, el carácter presuntamente opaco, estatutariamente ilegible de este tipo de textos se impone como una evidencia indiscutida, ya que la laboriosa producción del poeta-artesano se opone de un modo no menos sistemático al discurso exento de toda constricción del artista inspirado.

La escritura límpida y compleja de Bernardo Schiavetta inflige un desmentido radical a este prejuicio. Nada más claro, en efecto, que esta 
poesía preocupada por las reglas más clásicas y que genera un sentido inmediato, de cuya interpretación casi no cabe dudar. Pero nada más elaborado también que ese trabajo cuya lectura no se termina jamás, como lo demuestran claramente las reescrituras incesantes a las que el escritor somete sus propios versos, ya que la reciente transposición al francés sólo es por lo demás una manera de prolongar o amplificar la obra en su integridad, cualquiera que sea la lengua elegida.

El objetivo de este análisis es demostrar hasta qué punto simplicidad y complejidad, lejos de contradecirse o excluirse recíprocamente, establecen relaciones de complementariedad en estos poemas, y también analizar cuáles son sus consecuencias para la lectura propiamente dicha. Porque, leyendo a Schiavetta, se comprueba que la transparencia innegable de los juegos formales y de significación no conlleva en absoluto la interrupción o el agotamiento de la lectura, sino que permite, al contrario, la percepción de maniobras textuales siempre más sutiles; la prueba es que su examen preciso y riguroso acaba provocando - ¿forzosamente? - una reescritura, incluso a veces una serie de reescrituras del texto por el lector.

La complejidad no es evidente de golpe, sino que se desvela poco a poco, de modo que cada poema revela progresivamente un juego, es decir, la posibilidad de inscribir en el cuerpo de cada texto un conjunto de variantes pautadas; tan amplio resulta ser el abanico de estructuras, que se vuelven posibles por las leyes de estas composiciones.

Los distintos elementos de esta configuración pueden ser repertoriados desde la primera lectura del soneto titulado «...POE...» (Schiavetta, 1995: 78) ${ }^{1}$ :
...POE...
Me he disuelto en la nada que te ase
$Y$ he quemado los libros que no he escri
$Y$ no hay siquiera un párrafo erudi
Que me nombre en ninguna Encyclope
Me he disuelto en la nada, en la interme
Comarca de lo ausente y lo inaudi
Donde borro mi propio manuscri
Mi Ilíada, mi Eneida, Mi Come
Vuelvo hoy como la sombra de la so
Que entre los otros fui, que fui conmi
Pero al verme no tiemblas y no hu

1 Se trata del segundo poema de un ciclo de seis «sonetos de artificio». 
Sino que en un papel que no me no

Mi poeta, mi máscara, mi ami

Tú escribes mi poema y lo distru

La distancia en relación con la tradición hermética salta a la vista. En ningún momento puede ser considerado este texto como una ilustración de la poética de la alusión (Baetens, 1993) ${ }^{2}$, que se encuentra en el centro de todo hermetismo, bien porque el poeta recurra a un repertorio codificado cuya clave sustrae o esconde al lector indeseable, bien porque se obstina en reemplazar la designación que se creía demasiado directa por los encantos evanescentes de la sugerencia. Definitivamente, en Schiavetta el problema no viene del sentido del texto, que es transparente de principio a fin.

Al mismo tiempo, y es una segunda particularidad de «...POE...», el poema anuncia también su enorme apertura a la intervención creadora del lector. Más allá de su falta de cierre formal, de la que por supuesto tendremos que volver a hablar. «...POE...» es un texto plural, dispuesto a dejarse modificar de más de una manera. Sin embargo, esta apertura no está determinada por el diagnóstico de un defecto - debido a una falta de esmero en el momento de la elaboración del texto o a alguna imperfección material en su formalización, a los cuales quedan reducidas casi siempre las posibilidades de retoque lectural. La confrontación de dos formas no está basada en el deseo de oponer una «buena» forma a una «menos buena». Dicho de otro modo, esta confrontación no se hace en el marco de una investigación de tipo filológico o genético, donde se tiende muy a menudo a eclipsar una «lectio» por medio de otra (en efecto, no es raro constatar que la tradición material de un texto genera todo tipo de escorias, tanto en el nivel del manuscrito del mismo autor como en el de las repeticiones del texto en versiones infieles entonces); ni en la óptica de un examen estilístico, donde en más de una ocasión uno llega a descubrir las inevitables debilidades que quedan en un texto, por bueno que sea. (Como apuntó juiciosamente Borges, ningún texto es tan perfecto que no sea posible hacerle al menos una corrección.) Las modificaciones que se pueden aportar al texto de Schiavetta no conciernen el modo de la corrección: la reescritura no es una operación de maquillaje, de restauración, menos aún de reparación, sino, al contrario, una tentativa de hacer justicia a la complejidad generada por las estructuras mismas de la obra.

2 Para más detalles sobre los distintos empleos de esta figura estilística, véase Baetens (1993). 
Del mismo modo, la apertura a la pluralidad no es abandonada a la inspiración ni al capricho del lector. Como veremos, si hay, sin duda, varias maneras de reescribir la obra, el lector no es realmente libre para estrujar el texto en el sentido que le plazca dar a la escritura. Al contrario, la superación del poema sólo puede hacerse desde dentro de su propio programa, a la vez muy simple y muy complejo, y de su propio rigor, y aquí ninguna dimensión puede ser sacrificada.

No se trata entonces de reemplazar una versión por otra sino, aunque parezca imposible, de añadir una versión a la que ya existe y que no se deja eclipsar. Corolariamente, tampoco se trata de que la interpretación del lector sustituya a la letra del autor, sino de llegar al fondo de la lógica del texto cada vez.

\section{UNA AUSENCIA AMBIGUA}

Como ya hemos dicho, la singularidad más patente de este poema es el corte de la última sílaba de cada verso, más exactamente de todo elemento posterior a la última vocal acentuada, cosa que viene ya anunciada en los puntos suspensivos del título. No obstante, esta amputación resulta extraña por más de una razón.

Primero, la supresión despierta cierta curiosidad porque no conlleva aparentemente ninguna plusvalía composicional auténtica ${ }^{3}$. Como reconocemos sin problema el eslabón que faltaba y como este último se presta a una lectura en singular ${ }^{4}$, la decisión de ocultar una parte del texto sigue siendo un fenómeno de poca envergadura: es una insistencia retórica del tema poético, cuyas profundas implicaciones no están realmente valoradas.

Por otra parte, la censura despista porque no crea palabras nuevas en el seno de los términos así tratados, excepto «ase-» (de «asar», verso 1) y «come-» (de «comer», verso 8 ), combinación probablemente involuntaria cuyo humor dudoso entra tan poco en sinergia con el tono

3 Es cierto que la idea de sección está hábilmente puesta de manifiesto por la alineación a la derecha del texto, que permite así visualizar la «loncha». Pero este procedimiento se añade a la técnica de la apócope, sin ser realmente su motor.

${ }^{4}$ Las posibilidades de añadir sílabas parecen, en efecto, no admitir ningún margen de maniobra: asedia/escrito/erudito/Enciclopedia//intermedia/inaudito/manuscrito/Comedia//sombra/conmigo/huyes//nombra/amigo/distruyes. 
grave del texto que, en este sentido, apenas sería razonable profundizar en el campo endogramático del poema.

¿Deberíamos decir, por tanto, que la maniobra es gratuita? La comparación con el título arroja sobre el procedimiento una luz muy diferente. En el encabezamiento, efectivamente, el agotamiento de POE produce un endograma extremadamente significativo, lo cual obliga por lo menos a tomar en serio el texto tal como se halla dispuesto -negro sobre blanco-, y sobre todo a hacer una distinción categórica entre el núcleo del término, «POE», fuertemente subrayado, y su final, «...», un poco devaluado. Lo que es más, el nombre de POE, sobre todo en el contexto de los sonetos de artificio, es cualquier cosa menos arbitrario. A través de este autor se evoca también su Filosofía de la Composición, más precisamente las tesis relativas a la elaboración del poema hiperconstruido: recordemos brevemente que en esa obra se postula que todos los elementos de un poema deberían tender a un único y mismo objetivo cuyo secreto sólo es desvelado al final del texto. El elemento clausular se ve así violentamente proyectado a la zona delantera de la escena textual y más aún cuando tiene todo el aspecto de estar truncado.

Al ser sobrevaloradas y puestas en tela de juicio simultáneamente las palabras que sufren la elisión, y sobre todo la última palabra del texto, «distru/*yes» (de «destruir»), ocupan un lugar mucho más estratégico del que se había pensado a primera vista.

El análisis de los cortes quedaría, sin embargo, incompleto si no se ampliara a la aféresis responsable de la desaparición de las primeras letras de «...POE...». A priori, esta supresión es más difícil de rectificar que la ausencia de unidades al final de un sintagma. Allí donde, de hecho, «POE...» sólo puede leerse en buena razón como «poema» o «poeta», la diversidad de soluciones iniciales es, en cambio, mayor: «Antipoe...», «Al poe...», «Mi poe...», etc.

Ahora bien, como a primera vista no falta nada en el cuerpo del poema mismo, la pertinencia estructural de los puntos suspensivos al comienzo no se percibe inmediatamente. Mientras que hay una relación de equivalencia directa entre los tres puntos finales y el corte de las últimas letras de cada verso, tal simetría entre título y texto falla en los tres puntos iniciales. Como, a pesar de todo, la funcionalidad del corte final es bien real, el lector también es invitado a formular hipótesis en cuanto a la significación del corte inaugural, que podría significar entonces, por ejemplo, la presencia virtual de otros cortes en 
otros lugares del verso, hasta la posibilidad de una transformación aún más extensa del texto, por reescribir más que simplemente por completar. Las implicaciones de semejante punto de vista no tardarán en ponerse de relieve en la continuación de este comentario.

\section{VARIACIONES SOBRE UN SONETO}

Como «...POE...» tiene toda la apariencia de un soneto clásico, vamos a interesarnos primero por esta composición tradicional, y después por lo que se separa un poco de ella.

En cuanto a la arquitectura global del poema, la bipartición convenida entre cuartetos y tercetos no sólo es respetada, sino puesta vigorosamente en evidencia por la «volta», varias veces activada y significativa, al comienzo del verso 9. «Vuelvo» es efectivamente la única forma verbal colocada al principio de verso en todo el soneto. Es más, la raíz misma de este verbo mantiene con la anáfora muy subrayada «Me he disuelto» relaciones formales muy directas (los infinitivos son «disolver» y «volver», respectivamente). Finalmente, el valor metatextual o autorrepresentativo del término elegido no deja ninguna sombra de duda: para indicar el giro de un soneto, el verbo «girar» conviene estupendamente.

Por el contrario, en el esquema de las rimas se encierra una enorme sorpresa:

1 ase//dia

2 escri//to

3 erudi//to

4 Encyclope//dia

5 interme//dia

6 inaudi//to

7 manuscri//to

8 Come//dia

$9 \mathrm{so} / / \mathrm{mbra}$

$10 \mathrm{conmi} / \mathrm{go}$

$11 \mathrm{hu} / / \mathrm{yes}$

$12 \mathrm{no} / / \mathrm{mbra}$

$13 \mathrm{ami} / / \mathrm{go}$

14 distru//yes 
Efectivamente, mientras que las rimas idénticamente alternantes de género de los cuartetos corresponden a las reglas convencionales del soneto, sin embargo, ni las rimas alternantes, ni pareadas, ni alternantes de género de los tercetos (todas diferentes en el primer terceto, y luego repetidas por orden en el segundo terceto), pueden resultar sorprendentes al menos para el lector moderno. El resultado es forzosamente una insistencia en el tercer sonido de la rima, la forma «-yes» de los versos 11 y 14 , un poco inhabitual en cuanto a su distribución ${ }^{5}$, pero también en cuanto a su forma (en «...POE...» no hay ningún otro ejemplo de una rima en «E», ni de inclusión de una consonante final en la estructura de las palabras que riman).

El hecho de que al final de un soneto tan pautado descubramos elementos difíciles de prever, desconcertará a más de un lector. De hecho, muchas otras particularidades del mismo texto habrían podido prevenir a ese mismo lector acerca del estatuto ambivalente que en «...POE...» será el del último elemento de la estrofa. Si ampliamos el análisis a la estructura de las últimas sílabas impresas, encontramos las mismas características ya observadas en la estructura de las rimas sobrentendidas:

$\begin{array}{ll}1 & \text {-se } \\ 2 & \text {-cri } \\ 3 & \text {-di } \\ 4 & \text {-pe } \\ & \\ 5 & -m e \\ 6 & - \text { di } \\ 7 & -c r i \\ 8 & - \text { me } \\ & \\ 9 & - \text { so } \\ 10 & -m i \\ 11 & - \text { hu } \\ & \\ 12 & - \text { no } \\ 13 & -m i \\ 14 & - \text { tru }\end{array}$

Si la correspondencia de este esquema con el esquema estrófico y de rimas establecido a partir de las unidades implícitas se confirma global-

5 La estructura más común en los terceros contemporáneos es cdc/dee. En la época clásica, otras estructuras —como, por ejemplo, la de «...POE...»— no eran excepcionales. 
mente, la distinción de los cuartetos y de los tercetos más la acentuación de los últimos elementos de los tercetos pueden ser afinadas algo más.

En un primer momento, es cierto que la división entre cuartetos y tercetos se asemeja a un desequilibrio que beneficia sobre todo a los ocho primeros versos, claramente más regulares que los seis últimos. En efecto, mientras que encontramos por doquier rimas «ricas» ${ }^{6}$, las rimas leoninas o muy ricas parecen ser el privilegio casi exclusivo de los cuartetos -en todo caso en la parte impresa, explícita, del soneto. (Esa observación puede sorprender al lector español, que desconoce la distinción entre rima «normal» y rima «rica», pero la familiaridad del autor con el sistema francés, en que esta distinción es fundamental, obliga en nuestro análisis a subrayar la importancia de ese fenómeno fónico.) A esto hay que añadir que la homogeneidad del material fónico concernido por las rimas es mucho más profunda en los cuartetos que en los tercetos, donde resulta chocante la diversidad de los sonidos que riman. Finalmente, con la ayuda de estos rasgos que se combinan, el lector tiene la impresión de que las estructuras «perfectas», es decir, perfectamente simétricas, de los cuartetos se dislocan parcialmente en las dos últimas estrofas del poema.

Este análisis queda, sin embargo, un poco corto, ya que el soneto no se reduce al conjunto de sus rimas, explícitas o implícitas. A poco que se examine también el reparto de las estructuras sintácticas, de nuevo se identifica, sin duda alguna, la división de los cuartetos y de los tercetos, pero esta vez la vertiente más acentuada es indiscutiblemente la de los tercetos. Lejos de ser tratados como parientes pobres o de manera menos estricta que los cuartetos, estos últimos retienen la atención por el aumento de disposiciones formales de que han sido objeto. Una contracción o condensación absolutamente espectacular se observa efectivamente en todos los niveles.

En el plano macroscópico, la distribución de las proposiciones principales y de las relativas se organiza como sigue. En el primer cuarteto, a la repetición de una misma estructura (principal + relativa) en los dos primeros versos le sigue un fenómeno de escalonamiento y de amplificación, que hace que tanto la principal como la relativa ocupen ahora un verso entero. En el segundo cuarteto, este movimiento se

${ }^{6}$ N. de T. Rima «rica», en francés, es la que contiene al menos una vocal y su consonante de apoyo. El ejemplo que cita el diccionario Robert es: image/hommage. Conviene indicar, igualmente, que en francés rima «leonina» es la que se extiende a la sílaba anterior a la tónica: volant/consolant; animal/bien ni mal. 
refuerza todavía más, ya que la única principal y la única relativa rigen cada una dos versos enteros. Esta tendencia al ensanchamiento viene subrayada por el recurso a la yuxtaposición de complementos análogos, según un sistema binario en el verso 6 y terciario en el verso 8 . Dicho de manera esquemática («a» para oración principal y «b» para subordinada relativa):

$$
\begin{array}{ll}
1 & \text { ab } \\
2 & \text { ab } \\
3 & \text { a } \\
4 & \text { b } \\
& \\
5 & \text { a } \\
6 & \text { a } \\
7 & \text { b } \\
8 & \text { b }
\end{array}
$$

Los tercetos invierten esta lógica de expansión proporcional, por una parte, con la utilización sobre todo de las independientes, principales y relativas más cortas; por otra parte, con la subversión sutil del principio de la repetición de complementos colocados en los cuartetos. $\mathrm{La}$ alternancia y la longitud de las independientes y principales (simbolizadas unas y otras por «a») y de las relativas («b») obedece a este esquema (que elude en parte la serie de vocativos del verso 13 , relacionable tanto con el verso 12 como con el verso 14):

$$
\begin{array}{rl}
9 & \text { a } \\
10 & \text { bb } \\
11 & \text { aa } \\
& \\
12 & \text { b } \\
13 & ? ? \\
14 & \text { aa }
\end{array}
$$

Paralelamente al abandono de las técnicas de amplificación, se observa igualmente - por lo demás, lógicamente-, que el poema no suprime menos la repetición de sintagmas sintácticamente equivalentes, uno de los hechos más característicos de los dos cuartetos. Las dos relativas del verso 10 no realizan en absoluto la misma función. Una homonimia o, más exactamente, una diáfora comparable se reproduce en el verso 12, y de manera todavía más manifiesta, ya que al adver- 
bio comparativo «que» le sucede una forma parecida, que, de hecho, es un pronombre relativo.

El peor de los contrasentidos es que una mínima simetría dirija la construcción de los tercetos. Tanto en la segunda como en la primera mitad del soneto, están en funcionamiento convergencias extremadamente meticulosas, incluso si el régimen que es el suyo no es en absoluto el mismo. Los versos 1-8 responden esencialmente a una estructura lineal; los versos 9-14 presentan un ritmo y una organización mucho más «nerviosos», más cortados, y su simetría profunda sólo emerge en una lectura más detallada, más atenta a los detalles de la letra.

El análisis macroscópico aporta múltiples confirmaciones de estas observaciones. Es imposible que no resulte chocante el contraste entre, por un lado, las repeticiones largas e iniciales de los cuartetos, donde el primer hemistiquio del verso 5 repite literalmente, en una anáfora gigante, el primer hemistiquio del verso $1 \mathrm{y}$, por otro, las repeticiones mucho más cortas pero por contra más numerosas agazapadas tanto al principio como al final de los versos de los tercetos, primero en el nivel lexemático (con dos veces «sombra» en el verso 9, dos veces «que» en el verso 10 , dos veces «no» en el verso 11), luego en el nivel infralexical (con coyunturas, sobre todo en el segundo terceto, muy cercanas a ciertas sílabas: «no» en el verso 12 , «mi» en el verso 13 , eventualmente incluso «t(r)u» en los dos extremos del verso 14). Corolariamente, la toma en consideración de las unidades mínimas que son las letras permite reconocer en el encadenamiento fónico del verso 11, «tiembla/S Y NO/hu-», la exacta anticipación de la primera palabra del terceto siguiente, «sino», cuyo sentido sobrentendido ('sí', 'no') vuelve a formular además las ideas, en el momento en que se pasa de un terceto a otro, de oposición y de recuperación confundidas en la 'volta' situada en la transición de los cuartetos y de los tercetos.

Ya podemos concluir que la diferente elaboración de los cuartetos y de los tercetos no corrobora solamente la partición general sino relativa entre las dos partes del soneto, visible desde el comienzo del análisis, sino que induce sobre todo a hacerse más sensible a lo que separa, en el interior de cada una de las últimas estrofas, los dos primeros versos del último, e incluso a lo que distingue, en el interior del grupo formado por los versos 11 y 14, el verso final del primer terceto del verso final del soneto, ya que éste supone un problema mucho mayor que aquél en la composición del conjunto. 
Antes de extraer nuevas conclusiones, para después precisar lo que suponen esos recorridos sucesivos del poema para la cuestión de las relaciones entre escritura y lectura, es conveniente, sin embargo, imprimir un giro suplementario al análisis e inscribir las observaciones prosódicas, sonoras y lexicales en la estructura enunciativa del texto completo. Todo el poema sobredimensiona efectivamente la magnitud personal, intersubjetiva o, más exactamente, pronominal de la escritura.

¿Podemos seguir extrañándonos todavía de que el reparto de los principales morfemas pronominales siga de cerca los dispositivos ya señalados en más de una ocasión? En el verso 1, las posiciones extremas están ocupadas por el par «me»/»te», al aparecer ambos en una posición inacentuada infligida por la sinalefa. Por lo que se refiere a los cuartetos en general, las posiciones iniciales extremas, con la apertura de los versos 1 y 4 , luego 5 y 8 , se completan de manera simétrica, siempre con una forma ligada a la primera persona, ya que la desaparición provisional de la segunda persona en el segundo terceto viene señalada quizá por una inclusión de tipo clandestino, de nuevo en posición inacentuada, al final de la palabra misma que dice la ausencia: «ausente» (verso 6).

Tal y como cabía esperar, el primer terceto conlleva una inversión de esta situación. A la progresiva desaparición del «yo», que la diáfora excluye primero de la forma verbal «fui» (en el verso 10 es en un principio una primera persona y más tarde una tercera persona) y a la que la aglutinación priva después de toda autonomía léxica (en «Verme» del verso 11, la forma «-me» está unida al infinitivo), sigue una marcada reaparición de la segunda persona, incluso si el pronombre tónico no llega aún a emerger.

El segundo terceto, finalmente, primero atrae de nuevo toda la atención sobre la primera persona, pero ésta ha perdido su derecho a la posición-sujeto («me» es un complemento de objeto directo; «mi» un adjetivo posesivo). Por contra, en el pronombre tónico «tú», opcional en español, las desinencias bastan para marcar la persona verbal, que focaliza todas las miradas: es el único caso de un pronombre tónico en todo el soneto y surge además al principio del último verso de un poema enteramente compuesto con la vista puesta en su final.

Si tenemos en cuenta el cuidado con el que están imbricadas las diversas partes del soneto (el segundo cuarteto retoma anafóricamente el primero, los tercetos sustituyen a los cuartetos con ayuda de una 
«volta» subrayada en varias ocasiones, el final del primer terceto recoge ya los materiales fónicos de la apertura del último); si relacionamos igualmente las numerosas simetrías entre las posiciones extremas en los versos, en las estrofas, en el poema entero; si finalmente aceptamos leer los puntos suspensivos idénticos que preceden y siguen la palabratítulo como un comienzo o un indicio de circularidad, entonces se nos permitirá estrechar aún más las similitudes entre los versos 1 y 14 y postular la existencia de un inmenso quiasmo, velado por el corte de la sílaba final. Al par «me»/»te» del primer verso correspondería entonces, no los dos casos de la segunda persona («escribes/distruyes») que una primera lectura había actualizado sin ningún problema sino, aunque parezca imposible, y en este orden, una forma-tú, «escribes», y una forma-yo, que no puede ser otra que «distruyo».

De este modo la lectura cambia profundamente la materialidad del texto. Queda ahora por apuntalar con argumentos sólidos la modificación propuesta y después por reconsiderar lo que eso supone para la literatura hiperconstruida en general.

\section{YO, YO, YO}

A menos que quedemos indiferentes ante las articulaciones propias de un texto, cambiar una palabra dentro de él no es siempre cosa fácil. Muchos cambios que tendríamos ganas de operar sobre una escritura son, efectivamente, bien difíciles o imposibles, bien totalmente incompatibles con el espíritu o la letra del texto en cuestión, sobre todo en el caso de una obra tan trabajada como el presente «soneto de artificio». Tras demostrar que el nuevo término puede desempeñar realmente su papel, también es necesario preguntarse qué ganancia auténtica representa.

En «...POE...» la forma «destruyo» parece sólo el efecto de una cierta licencia poética. El salto de la segunda a la primera persona transgrede las reglas habituales de la correferencia en español, que suponen que dos formas verbales coordinadas por «y» - la primera es introducida aquí por el pronombre tónico «tú», la segunda libre de todo cambio de sujeto explícito- tienen el mismo referente. Al leer «destruyo» en lugar de «destruyes», lo cual es posible gramaticalmente hablando pero nada probable, restituimos de hecho doblemen- 
te la primera persona: en cuanto se cambia la terminación, la fuerza del contraste entre segunda y primera persona es tal, que implícitamente el lector se ve obligado a añadir el pronombre tónico «yo». Los puntos suspensivos iniciales verifican quizá en este caso su razón de ser fundamental: podrían sugerir, en efecto, no la presencia callada de un término desaparecido, sino la posibilidad de añadir un término no previsto en la cuenta inicial. La licencia poética perdería así mucho de su arbitrariedad.

Que se gane mucho incluso con la lectura «destruyo», se puede especificar por lo menos de dos maneras. Primero, la forma «destruyo» se integra perfectamente bien en las otras estructuras del poema, por ejemplo con las rimas (gracias a «-yo», la cohesión sonora y prosódica de los tercetos crece notablemente).

En segundo lugar, es incontestable que la supresión final de la sílaba -yo, al final de un texto consagrado a la desaparición elocutiva del poeta, concuerda de maravilla con el sentido mismo del conjunto: la exclusión del lexema «yo» es una perfecta transposición literal de la evaporación del yo anunciado desde las primeras palabras del poema. La idea de un yo «tachado», que es el sentido esencial del poema, se expresa más adecuadamente por medio de «destruyo» que de «destruyes». Si es verdad que el poema se presenta como una variación sobre la paradoja de la autodesaparición, que es a su vez una variante de la paradoja de la negación, la proscripción material del «yo» al final del texto es una elección lógica: así como, para negar algo, debo comenzar ineluctablemente por plantear su existencia, de igual modo sólo puedo decir o escribir que desaparezco de mi poema diciéndolo o escribiéndolo en un poema, y la suspensión última del pronombre «yo» es la condensación perfecta de este mecanismo.

Si no faltan buenas razones para leer «destruyo», el asunto podría no terminar aquí.

\section{NO HAY YO SIN TI}

Cuestiones más profundas se plantean en relación con el último verso. No conciernen únicamente al derecho que tienen los lectores a transformar el objeto sin el cual nada sería posible, sino también y sobre todo a las modalidades más generales de este procedimiento. El 
cambio propuesto va efectivamente más lejos que el derecho a la interpretación personal que en nuestros días ha sido concedida a todo lector por la benevolente doxa crítica en literatura. Por lo demás, la hipótesis de lectura que supone «destruyo» no llega a tanta libertad interpretativa, en la medida en que apenas es el resultado de un acto hermenéutico, sino, muy al contrario, la consecuencia del desciframiento de una red de estructuras en la que la opinión del lector individual no se tiene en cuenta de modo alguno. De hecho, para el significado mismo del poema, la transformación examinada se parece extrañamente a una operación blanca, ya que la «lección» del nuevo verso 14 no aporta nada verdaderamente nuevo en relación con lo que ya se había dicho, por ejemplo, en el verso 7.

Todo el interés del texto está justamente en no conducir a un sentido nuevo, de manera que la nueva forma puede ser juzgada en sí misma, sin interferencia con los problemas siempre espinosos de la interpretación de un sentido. «...POE...» induce la formulación de un término nuevo, rival del primero y no sustitutivo.

Entre otras muchas cosas, esta operación significa que reemplazar «distruyes» por «distruyo» es tanto perder como ganar, es decir, borrar el texto de la segunda persona en provecho exclusivo del infratexto de la primera persona, que daría por lo tanto la «clave» del sintagma rechazado. Al borrar «destruyes» y dejar sólo «destruyo», la lectura desembocaría en un empobrecimiento y no en una mejora del texto. En efecto, las numerosas torsiones y complicaciones que engendran un contraste tan sorprendente entre cuartetos y tercetos, no dejarían de ser englutidas por la censura de las formas de la segunda persona, cuyo lugar, en todo el proceso de lectura que hemos tratado de reconstituir, es absolutamente ineludible.

La aproximación plural y ecuménica del último verso explica también - y esto es capital- que la producción lectural de la forma «destruyo» no obedece en nada a la lógica binaria de elección entre la buena y la mala lectura. Lo que cuenta en «...POE...» es la sobreimpresión de las lecturas, no su rivalidad o su saqueo recíproco.

En resumen, si en la literatura hiperconstruida el cambio de una palabra es todo menos un hallazgo, puesto que es de rigor que el término nuevo materialice una de las posibilidades estructurales reconocidas por el análisis detallado del texto, el cambio en cuestión no es ni siquiera un cambio propiamente dicho, ya que la primera versión no debe ser excluida en beneficio de la nueva. Es más: ni siquiera se trata 
quizá de una transformación sin más, ya que la modificación propuesta aparece sobre todo como la continuación fiel de la actividad misma del texto, que orienta al lector hacia este tipo de intervenciones. El texto de Bernardo Schiavetta admite su alteridad por la misma razón y con la misma fuerza que su propia estructura.

Otras distinciones se podrían añadir aún, como por ejemplo la que opone este tipo de escritura a la estética contemporánea del clinamen 7 . Pero, vista la complejidad de estos nuevos problemas, esta discusión sólo podría ser objeto de otro estudio.

\section{Referencias bibliográficas}

BAETENS, J. (1993). «L'allusion, de la poétique à l'idéologie». Cahiers marxistes 168.

- (1995). L'éthique de la contrainte. Leuven: Peeters.

SCHIAVETTA, B. (1995). Con mudo acento. Albacete: Barcarola.

7 El clinamen consiste en introducir en un dispositivo muy pautado una cierta cantidad de irregularidades cuyo objeto es romper el proceso mecánico y monótono de ciertos textos escritos bajo constricción. Para una discusión de ciertos empleos oulipianos del clinamen, véase Baetens (1995). 